\title{
Genome-Wide Association Study (GWAS) Reveals The Genetic Basis of Brace Root Angle and Diameter in Maize
}

Daqiu Sun

Liaoning Province Research Center of Plant Genetic Engineering Technology, Shenyang Agricultural University

Sibo Chen

Shenyang Agricultural University, Ministry of Education

Zhenhai Cui

Liaoning Province Research Center of Plant Genetic Engineering Technology, Shenyang Agricultural University

Jingwei Lin

Liaoning Province Research Center of Plant Genetic Engineering Technology, Shenyang Agricultural University

Meiling Liu

Liaoning Province Research Center of Plant Genetic Engineering Technology, Shenyang Agricultural University

Yueting Jin

Liaoning Province Research Center of Plant Genetic Engineering Technology, Shenyang Agricultural University

Ao Zhang

Liaoning Province Research Center of Plant Genetic Engineering Technology, Shenyang Agricultural University

Yuan Gao

Liaoning Province Research Center of Plant Genetic Engineering Technology, Shenyang Agricultural University

Huiying Cao ( $\nabla$ chy@syau.edu.cn)

Liaoning Province Research Center of Plant Genetic Engineering Technology, Shenyang Agricultural University

Yanye Ruan

Liaoning Province Research Center of Plant Genetic Engineering Technology, Shenyang Agricultural University

\section{Research Article}

Keywords: Maize (Zea mays), Brace angle, Brace diameter, GWAS, Genetic architecture, SNPs

Posted Date: October 11th, 2021

DOI: https://doi.org/10.21203/rs.3.rs-846593/v1

License: (c) (1) This work is licensed under a Creative Commons Attribution 4.0 International License. Read Full License 


\begin{abstract}
Background:Brace roots are an important part of the maize root system. Among brace root traits, brace root angle (BRA) and brace root diameter (BRD) are important components that affect plant growth and development. However, there are no reports on the genetic basis of maize BRA and BRD.
\end{abstract}

Results:Here, a genome-wide association study (GWAS) was conducted using 508 associated populations with extensive natural variation. The broad heritability of BRA and BRD reached 0.91 and 0.82 , respectively. The analysis of different subgroups showed that there were significant differences in BRA traits in different subgroups, whereas there was no significant difference in BRD. Evaluation of phenotypic diversity in three different environments showed that BRA and BRD exhibit a wide range of natural variability. In the GWAS, the BRA and BRD were combined with 55,8629 single nucleotide polymorphisms, and four candidate genes were found for BRA within the threshold of $\mathrm{P}<1.78 \times 10^{-6}$ that were significantly related to BRD). These genes may (1) participate in maize brace root cell wall synthesis through cell transport (GRMZM2G479243); (2) involve hormone signaling pathways in the horizontal expansion of brace root cells (GRMZM2G101928 and GRMZM2G174736); or (3) involve the PLETHORA (PLT1/2) gene (GRMZM2G151934) to promote stem cells and transport expanded cells to affect the growth of root meristems.

Conclusions:These results provide theoretical information for understanding the genetic basis of brace root development. Further research on candidate genes will help clarify the molecular pathways regulating BRA and BRD in maize.

\title{
Background
}

The maize root system, as the main organ supporting the above-ground part of the plant, can seriously affect the lodging resistance of maize. Maize root lodging resistance will greatly improve the yield of grain or dry matter and the quality of plant harvest[1]. The maize root system is composed of main radicle and lateral radicle. It participates in the absorption of water, $\mathrm{N}$, and other mineral nutrients[2], synthesizes physiologically active substances, and improves the lodging resistance of roots. Sex[3-9] under drought conditions, the maize root system has a partial impact on maintaining grain yield and providing anchorage in the soil[10,11]. The postembryonic root system is composed of continuous bud nodes and lateral roots. The bud roots or node roots formed in the ground are called crown roots, and those formed on the ground are called brace roots[10]. The brace roots of maize appear from continuous stem nodes, and their contribution to the development of maize plants depends on their related traits. These traits consist of the tier number of brace roots (TN), the number of brace roots (RN), the radius of brace roots (RBR)[12], brace root fresh weight, and brace root dry weight[13], among others. Maize brace roots can improve lodging resistance and can also have a significant impact on grain yield by absorbing nutrients and water under limited soil water conditions[4, 14, 15]. Root lodging is related to brace root traits, such as root volume[16], number of upper internodes[17], root diameter[18], and number of internodes[17]. In particular, the strength of plant anchoring is determined by the number, diameter, and root angle of brace roots[19,20]. This in turn affects the lodging resistance of the plant.

Studies have shown that plants with higher phosphorus acquisition efficiency exhibit a higher root-to-stem weight ratio[21, 22], reduced root diameter[23], longer and denser root hairs[11], and increased lateral roots[24]. In addition, brace roots are different to crown roots, and the molecular mechanisms affecting brace root development are still poorly understood[25]. The maize $A P 2 / E R F$ transcription factor decreased the number of brace roots by inserting a mutant RAP2.7-Mu into ZmRAP2.7, thereby revealing the function of ZmRAP2.7in brace root development. Multi-drug and toxin extrusion (MATE) transporters may lead to the development of brace roots[26].

Genome-wide association analysis, also known as linkage disequilibrium mapping (LD mapping) or association mapping, is based on linkage disequilibrium, and the materials used must contain a wide range of natural mutations. The analysis method of finding the target trait and the gene locus that controls the trait has been widely used in genetic mechanism analysis of complex quantitative traits[27-30]. At present, the genetic basis of maize brace root grip radius, brace root layer number, and quantitative traits of brace roots[12] has been analyzed through GWAS. In addition, aranzana successfully found key genes that affect the flowering period and control disease resistance in maize using GWAS analysis[31]. Cui analyzed the genetic structure of husk length, Husk layer number, husk width, and husk thickness using an inbred line composed of 508 related populations with extensive natural variation[12, 32]. However, the genetic basis of BRA and BRD has not yet been studied.

The brace root angle and diameter used in this study are quantitative traits and are regulated by multiple genes. Under three environments, 508 maize inbred lines with 558,629 SNPs genotypes were subjected to GWAS analysis. The purpose of this study was to analyze BRA and the phenotypic diversity and genetic basis of BRD. This study also identified a series of candidate genes related to BRA and BRD, which provided a beneficial resource for further functional research.

\section{Results}

\section{BRA and BRD diversity and broad sense heritability Diversity and broad heritability of BRA and BRD}

The phenotype data of 508 inbred lines were measured independently in Shenyang City, Liaoning Province, in 2016 and 2017 and Siping City, Jilin Province, in 2016, and the phenotypic values of three environmental field trials were used as random effects to calculate the BLUP values (Supplementary Table S1). In the three environments, the average heritability of BRA and BRD was about $0.91 \%$ and $0.82 \%$, respectively (Table 1 ). 
Table 1

Brace root angle, diameter diversity and generalized heritability

\begin{tabular}{|c|c|c|c|c|}
\hline BR Trait ${ }^{\mathrm{a}}$ & Source of variation ${ }^{b}$ & F value & Significance $^{c}$ & $\mathrm{H}^{2 \mathrm{~d}}$ \\
\hline \multirow[t]{3}{*}{ BRA } & Environment $(\mathrm{E})$ & 1062.3195 & $<0.01^{\star \star}$ & \multirow[t]{3}{*}{0.91} \\
\hline & Genotype(G) & 968.9211 & $<0.01^{\star *}$ & \\
\hline & $\mathrm{G} \times \mathrm{E}$ & 81.5994 & $<0.01^{* *}$ & \\
\hline \multirow[t]{3}{*}{ BRD } & Environment(E) & 0.0205 & $<0.01^{* *}$ & \\
\hline & Genotype(G) & 0.0199 & $<0.01^{\star *}$ & 0.82 \\
\hline & $\mathrm{G} \times \mathrm{E}$ & 0.0034 & $<0.01^{\star *}$ & \\
\hline \multicolumn{5}{|c|}{ a BRA brace root angle, BRD brace root diameter } \\
\hline \multicolumn{5}{|c|}{${ }^{\mathrm{b}} \mathrm{G}$ and $\mathrm{E}$ indicate genotype and environment, respectively, and $\mathrm{G} \times \mathrm{E}$ indicate interaction of $\mathrm{G}$ and } \\
\hline \multicolumn{5}{|c|}{${ }^{c *}$ Significant at $P \leq 0.05 ;{ }^{* *}$ Significant at $P \leq 0.01$} \\
\hline
\end{tabular}

\section{Correlation between BRA and BRD}

As shown in (Fig. 1), the angle and diameter of brace roots all show a continuous normal distribution with a slight left skew. The BRA distribution ranged from $43.15^{\circ}$ to $63.97^{\circ}$, and the BRD distribution ranged from 0.39 to $0.52 \mathrm{~mm}$. The BRA and BRD were significantly correlated, and the correlation coefficient reached 0.27 .

The correlation between the angle and diameter of brace roots and the other 16 agronomic traits is shown in (Fig. 2). Among them, plant height (PH), ear height (EH), ear position leaf length (ELL), tassel maximum axis length (TMAL), tassel branch number (TBN), and leaf number above ear (LNAE) belonged to morphology learning indicators. The powdering stage (DTA), spinning stage (DTS), and heading stage (DTH) belonged to the characteristics of the growth period. Ear length (EL), ear diameter (ED), cob diameter (CD), kernel number per row (KNPR), grain weight (GW), cob weight (CW), and kernel width (KW) were yield traits. The brace root angle was significantly positively correlated with ear length (EL), with a correlation of 0.16 . Brace root angle and ear diameter (ED) were significantly positively correlated, with a correlation of 0.13 . Brace root diameter and kernel width (KW) were significantly positively correlated, with a correlation of 0.16 .

\section{Population structure, BRA and BRD of four major subgroups and different environments}

From the violin diagram of BRA of the four major subgroups (Fig. 3-a), it can be seen that brace root angle traits were significantly different in different subgroups, and the mean brace root angles in SS and TST subgroups were significantly lower than those in the average MIXED and NSS subgroups, indicating that population structure had a certain influence on this trait. As seen in the violin diagram of BRD of the four major subgroups (Fig. 3-b), distributions of the MIXED, NSS, and SS subgroups were relatively similar, while the distributions of TST subgroups were special and widely distributed. In addition, there were no significant differences in BRD among the four subgroups, indicating that the population structure had no significant effect on this trait. There were significant differences in the angle and diameter of brace roots in different environments (Fig. $<$ link rid="fig3" $>3</$ link $>-c$ and 3-d).

\section{Genome-wide association analysis}

To minimize the effect of environmental variation, phenotypic BLUP values across three environments were used for association studies. GWAS was performed using a mixed linear model (MLM) and both kinship relation- ship (K matrix) and population structure (Q matrix) were taken into account to avoid spurious associations. In total, we identified 2 and 3 SNPs significantly associated with BRA and BRD, respectively (Fig. 4; Fig. 5; Table 2). The percentage of phenotypic variation explained by the identified SNPs $\left(R^{2}\right)$ for BRA and BRD were $1.9 \%$ and $14.68 \%$, respectively (Table 2 ). 
Table 2

SNPs chromosome positions and candidate genes significantly correlated with BRA and BRD identified by GWAS using the MLM method

\begin{tabular}{|c|c|c|c|c|c|c|c|c|c|}
\hline Traits & SNP & Chr & Positions(bp) & Allell $^{\mathrm{a}}$ & P-value & $\begin{array}{l}R^{2} \\
(\%)^{b}\end{array}$ & Gene & Gene interval (bp) ${ }^{d}$ & Annotatione \\
\hline BRA & chr10.S_4090245 & 10 & 4090245 & $\mathrm{G} / \mathrm{A}$ & $\begin{array}{l}5.36755 \mathrm{E}- \\
07\end{array}$ & 1.90 & GRMZM2G101928 & 4159744.4166605 & $\begin{array}{l}\text { Zinc induced } \\
\text { facilitator-like } \\
1\end{array}$ \\
\hline
\end{tabular}

$\begin{array}{ll}\text { Total }^{\mathrm{C}} & 1.90\end{array}$

\begin{tabular}{|c|c|c|c|c|c|c|c|c|c|}
\hline & & & & & U & & & & protein 2 \\
\hline & & & & & & & GRMZM2G479243 & 140608664.140619439 & $\begin{array}{l}\text { Leucine-rich } \\
\text { repeat protein } \\
\text { kinase family } \\
\text { protein }\end{array}$ \\
\hline BRD & chr10.S_95437751 & 10 & 95437751 & $\mathrm{~A} / \mathrm{C}$ & $\begin{array}{l}1.71503 \mathrm{E}- \\
06\end{array}$ & 3.95 & GRMZM2G174736 & 95447060.95453186 & $\begin{array}{l}\text { hydroxyproline- } \\
\text { rich } \\
\text { glycoprotein } \\
\text { family protein }\end{array}$ \\
\hline
\end{tabular}

${ }^{a}$ Major/minor alleles, underlined bases indicate favorable alleles

${ }^{b}$ Percentage of phenotypic variation explained by the cumulative effect of a single significant SNP

${ }^{\mathrm{c}}$ Total percentage of phenotypic variation explained by all significant SNPS

${ }^{d}$ Position according to the B73 reference sequence (version 2)

e Name of the homologous gene in Arabidopsis thaliana or rice

\section{Expression patterns of candidate genes in different maize tissues}

Candidate gene expression analysis is conducted through the analysis of the FPKM value of the candidate gene on the Maize GDB website, and the statistical analysis results were developed in the form of tissue-specific expression heat maps. Four candidate genes related to BRA and BRD were identified through MLM (Table 2). Among them, four candidate genes were divided into eight functional types: anabolic pathways, protein binding, hormone signaling pathways, transport regulation, DNA binding, cell transport, structural proteins, and unknown functions (Supplementary Table S2). Among them, four candidate genes were significantly related to BRA and BRD (Table 2). To determine whether genes represented by significant SNPs were specifically expressed in brace root angle and diameter tissues, the published RNA-Seq data set was used from tissue-specific expression heat maps of candidate genes compiled in 11 different organs/tissues, including brace root tissues (Fig. 6). Among them, there were two candidate genes. Compared with other tissues, GRMZM2G479243 and GRMZM2G174736 had a lower expression trend in brace roots, while GRMZM2G151934 and GRMZM2G101928 had a higher expression trend in brace roots compared with other tissues. The high and low expression levels of these candidate genes in brace roots further indicate their relevance to brace roots.

\section{Discussion}

\section{Novel revealing the genetic basis of BRA and BRD}

The heritability of maize brace root-related traits has been reported; the heritability of brace root layer (TN), brace root number (RN), and brace root grip radius (RBR) ranged between 0.69 and $0.80[40]$. In this study, BRA and BRD showed extensive phenotypic variation and followed a normal distribution, and showed a very significant positive correlation ( 0.27 ; Fig. 1$)$, indicating that brace root traits are quantitative traits. Under multi-gene regulation, there may be co-regulated gene loci. Genetic analysis showed that BRA and BRD had high heritability ( $h^{2}=0.91$ and 0.82 , respectively; Table 1 ), indicating that the quantitative trait is suitable for GWAS[41]. In the three environments, the genotypic effects were significant for BRA and BRD, demonstrating that these genes were involved in the control of BRA and BRD (Table 1). The environment $\times$ genotype interaction also had a significant impact on BRA and BRD, which indicated that the degree of transmission of BRA and BRD in maize was different from one location to another (Table 1). In other words, the genetic effects of BRA and BRD involved in inheritance were different depending on the environment. Therefore, different lines can be selected for specific environments to improve BRA and BRD in breeding projects.

\section{Analyzing correlation between maize BRA, BRD and other agronomic traits}

As a component of the root system of maize, brace roots can not only play a supporting role but also have a certain impact on yield traits. As shown in (Fig. 2), BRA has a significant positive correlation with ear length (EL) and ear diameter (ED), with correlation coefficients of 0.16 and 0.13 , respectively. BRD had a significant positive correlation with grain width (KW), with a correlation coefficient of 0.16 . As an important nutrient, $N$ seriously affects the growth and development of maize plants. Studies have shown that nitrogen uptake by plants affects the number, diameter, angle, volume, and dry weight of brace roots[42]. The angle of maize canopy roots and brace roots was positively correlated with rooting depth. As the rooting depth increases, the plant's ability to absorb nitrogen increases and the more nitrogen it absorbs[43]. Nitrogen can effectively provide the nutrients required by maize, and higher nitrogen utilization 
can increase the dry matter quality of maize plants and grain yield and provide energy for the growth and development of maize. The indicators of maize growth and development include the number of ear rows, the number of grains, ear length, and ear thickness. Therefore, by affecting the angle of brace roots, the ear length and ear thickness can be further affected in maize[44, 45]. The diameter of brace roots is highly correlated with brace root fresh weight ( $r=0.730$ ) and brace root dry weight $(r=0.729)[13]$. The increase in fresh weight and dry weight of brace roots directly affects the nitrogen uptake by maize brace roots[42], and nitrogen uptake will increase grain yield by affecting the increase in sucrose and grain starch content[46]. Grain width is an important indicator of grain yield traits[47], as explained by the significant positive correlation between brace root diameter and grain width (0.16).

\section{Changes in the angle and diameter distribution of brace roots among the four major subgroups}

Maize originated in the tropics and was then domesticated in subtropical and temperate regions. Therefore, the morphological structure of maize is strongly influenced by population structure[48]. To study the influence of population structure on BRA and BRD, the phenotypic variation of maize BRA and BRD between different subgroups was compared (Fig. <link rid="fig3">3</link>-a and 3-b). As shown in (Fig. 3-a), there were significant differences in brace root angles among the four subgroups. The two subgroups of NSS and SS had a similar distribution of brace root angles. The average and scale of brace root angles of the two subgroups of MIXED and TST increased, and the two subgroups of NSS and SS were comparable to the two subgroups of MIXED and TST.

There were significant differences in brace root angles, indicating that the brace root angles of maize inbred lines of tropical and subtropical origin had a wider distribution range. This may be due to the external environment. To prevent the excessive evaporation of water from maize plants due to the high temperature in tropical regions, natural selection prefers maize brace roots with a wide range to extract more water. In contrast, due to the relatively low temperature in temperate regions, transpiration is relatively weak, and generally, the angle of brace roots has a small distribution range. Therefore, maize from temperate regions tends to have inbred lines with more concentrated angles. As shown in (Fig. 3-b), there was no significant difference among the four subgroups of brace root diameter. During the evolution of maize, the trait genes that control the diameter of the brace roots were distributed in each subgroup after a high degree of recombination, and during the long domestication process, the trait genes were less affected by genetic relationships. In addition, the angle and diameter of brace roots show significant differences in different environments, indicating that there is a wide range of natural variations (Fig. <link rid="fig3">3</link>-c and 3-d).

\section{Role of genes specifically expressed in brace roots causing morphological changes classification and pathways of candidate genes involved in BRA and BRD}

Brace roots can not only improve lodging resistance but also have a significant impact on grain yield by absorbing nutrients and water under limited soil moisture conditions $[4,14,15,49]$. However, the genetic basis of BRA and BRD is still unclear. In this study, the MLM method was used to identify four genes that were significantly related to BRA and BRD (Fig. 6). Functional annotations indicated that these candidate genes were mainly placed in several functional groups, such as DNA binding, hormone signaling pathways, and cell transport.

The plant cell wall is a dynamic structure, and its change is a response to the external environment. Two Leu-rich-like receptor kinases (FE/1 and FEI2) have been identified in Arabidopsis thaliana with a high degree of interaction with plant cell wall synthesis. The $F E /$ system can process the turgor signal transmitted by the SOS5 system and stimulate cellulose synthase to synthesize the cell wall. The gene mutations of FEI 1 and FEI lead to the break of the signal transmission chains, and plants sense the changes in the external environment and its biological effects on cellulose. Synthesis also plays a role in inhibition[50]. Debarati Basu (2016) compared the growth indicators of $F E /$ mutants in different environments and found that compared with wild-type Arabidopsis thaliana, the hypocotyls of $F E /$ mutants had shorter radicles and a larger diameter[51]. Fluorescent protein staining showed that $F E /$ had a higher expression in root tips. FEl1 was highly expressed in the specific expansion of Arabidopsis root cells. GRMZM2G479243 is highly homologous to the FEI coding gene $A T 1 G 31420.2$, which is predicted to play a role in the synthesis of brace root cell walls in maize.

The morphogenesis of plant organs depends on the intercellular flow of plant hormones, and directional signal transduction is determined by the polar subcellular localization of the auxin transporter synthesized by the PINFORMED (auxin export carrier PIN) gene[52]. The auxin synthesized from the brace roots is transported to the roots via vascular tubes. After auxin transport reaches the root tip, it is redistributed to the elongation zone of the root system. At this time, IAA enters the cell through the AUX1 carrier in the form of auxin, then enters the cell through the PIN carrier, and finally exports in the form of ions[53]. During this process, auxin is unevenly distributed on both sides of the elongation zone under the influence of gravity, and a geotropic reaction occurs. The phosphorylation kinetics of the PIN protein are affected by protein phosphokinase PP2A and PINOID, and the ROTUNDA3 (RON3) protein, as a regulator of PP2A phosphokinase, can play a role in regulating PP2A activity [54]. RON3 is a unique higher plant-specific gene. The map location technology identified 18 genes, including $A T 4 G 24500$, that were significantly associated with RON3, which encodes a proline-rich protein and phenotypes the RON3-1 mutant. The identification showed that the number of lateral roots at the seedling stage was significantly reduced, and geotropic growth, which is affected by gravity, was significantly weakened[55]. Furthermore, Zhan (2012) identified AT4G24500 as the SIC gene, which encodes a hydroxyproline-rich protein[56]. The SIC gene is a functional gene necessary for the plant to maintain normal growth and development. The phenotype of $S / C$ mutants was identified. It showed a variety of developmental defects, including reduced plant height, delayed flowering, increased leaf edge serrations, and abnormal roots. In maize, GRMZM2G174736 is a homologous gene of AT4G24500. Therefore, GRMZM2G174736 may play a role in the polar transport of auxin and affect the morphogenesis of maize roots.

Polar transport of the phytohormone auxin is the key to regulating plant growth and development. Polar transport of auxin in Arabidopsis roots requires the action of the MFS transporter, which is zinc-induced promoter-like 1 (ZIFL1). Reverse genetics showed that the ZIFL1.1 transporter regulates various root growth hormone-related processes and may indirectly regulate growth through cytokines used in the process of auxin transport; it may act by regulating the abundance of PIN2 in the plasma membrane of the root tip[57]. Auxin regulates various unrelated processes by directing cell division and expansion, such as embryo, root, and vascular bundle formation, post-embryonic organogenesis, and geotropism[58]. The chemical growth hypothesis better describes the basis for the movement of auxin cells, in which the proton gradient is mainly generated by the plasma membrane $\mathrm{H}^{+}$-ATPases between the neutral cytoplasm and 
the outside of acidic cells to drive the uptake and efflux of auxin[59]. This hypothesis presumes that there are local auxin influx and efflux carriers in the plasma membrane; thus, the asymmetric positioning of the coupling between adjacent cells provides directionality for cytokine flow[60]. In addition, auxin can also control the growth of Arabidopsis roots by regulating the cellular response to GA[61]. Therefore, GRMZM2G101928, as a homologous gene of AT5G13750.1, may affect the horizontal expansion of maize brace root cells and then affect morphogenesis.

Root growth and development is controlled by root tip meristems, and the size of root tip meristems affects the size of roots. In this study, GWAS was used to identify a candidate gene related to BRD traits and DNA binding, GRMZM2G151934, whose DA1 homologous protein 2 (DA1-related protein 2, DAR2) can regulate the size of root apical meristems. Relevant studies have shown that cytokinin and auxin antagonism can affect cell proliferation and differentiation, thereby regulating the size of roots by affecting the abundance of SHORT HYPOCOTYL2 (SHY2/IAA3). SHY2 affects the distribution of auxin in root meristems by inhibiting the auxin-induced expression of the PIN-FORMED (PIN) auxin transport gene. The PLETHORA (PLT1/2) gene affects the growth of root meristems by promoting stem cells and transporting and expanding cells[62]. In addition, DA1-related protein 2 (DAR2) acts downstream of cytokinin and SHY2 but upstream of PLT1/2 to affect the size of the root meristem[63]. Therefore, GRMZM2G151934, as a homologous gene of AT4G24500.1, may play a role in the growth of brace roots and affect the morphogenesis of maize roots.

\section{Conclusions}

This study revealed the genetic structure of maize BRA and BRD and the mechanism of controlling natural variation through GWAS. The 558,629 SNPs markers from 508 inbred line genotype populations around the world showed extensive variation. Studies have shown that BRA and BRD traits have a high level of inheritance. In addition, the four candidate genes of BRA and BRD may be involved in maize brace root cell wall synthesis, lateral expansion of brace root cells, cell transport, DNA binding, and the hormone signaling pathway. Candidate genes provide valuable resources for further dissecting the molecular network that regulates BRA and BRD in maize. The identification of SNPs will help promote the marker-assisted selection of BRA and BRD in maize breeding programs.

\section{Materials And Methods Plant material}

The genome-wide association population consisted of 508 inbred lines with extensive polymorphisms. Among them, there were 60 inbred lines from the American Maize Germplasm Innovation Project, 223 inbred lines from the International Center for Maize Improvement (CIMMYT), and 225 inbred lines from Chinese germplasm resources. All materials has preserved College of Biological Science and Technology, Shenyang Agricultural University. Most of the inbred lines from CIMMYT were derived from tropical or subtropical regions, and most of the inbred lines from the United States and China were of temperate origin. The genome-wide related population was clearly divided into four subgroups: the SS subgroup had 27 inbred lines; the NSS subgroup had 70 inbred lines; the TST subgroup had 196 inbred lines; and the remaining 215 inbred lines were divided into MIXED subgroups[33, 34]. SNPs data from maize SNP50Beadchip and RNA-Seq developed by Illumina provided the genotypes for all inbred lines, with a total of 558,629 SNPs markers. Each SNP marker underwent quality testing, and unqualified SNPs were recorded and deleted[33].

\section{Field trial}

The 508 inbred lines of the genome-wide association group were grown in three environments in China: Shenyang City, Liaoning Province (SY) (123 $25 \mathrm{E}$, $41^{\circ} 48 \mathrm{~N}$ ) in 2016 and 2017, and Siping, Ji lin Province, in Northeast China in 2016. All lines were planted in a randomized complete block design and repeated twice. Each inbred line was planted in a single row, with a length of 3 meters and a width of 0.67 meters, with a 0.4-meter aisle in the middle.

\section{Method}

The angle and diameter of the root system were investigated 30 days after planting. At this time, the brace roots were in good condition without cracking or wilting, and the measured brace root traits were more accurate, improving the accuracy of the measurement and facilitating subsequent correlation analysis. Measurement selection was based on the first level of the root system from top to bottom. Two plants with similar growth were selected for measurement in each row. BRD was measured in the middle of the brace roots using an electronic vernier caliper (mm). BRA was measured between the occurrence point of brace roots and the main stem using a protractor (degrees).

\section{Statistical analyses}

The mixed linear model (MLM) was used to calculate the analysis of variance of the brace root traits of maize in related populations: $y i j k=\mu+e_{l}+r k(I)+f i+(f e) i l$ $+\varepsilon$ lik, where $\mu$ represents the total average phenotype, and $f i$ is the first "i genotype effect" of the "family," e, represents the " $l$ " environmental effect, ( $f e$ ) $i$ i is the interaction effect between the " $r$ " family and the " $"$ " environment $r k$, $(I)$ is the repeated influence within the environment, and $\varepsilon$ lik is a random error. The "PROCMIXED" program in SAS software was used to analyze the phenotypic variation of maize brace root traits. The Best Linear Unbiased Prediction (BLUP) of maize brace root traits also used a mixed linear model, and the average value was added to the estimated value to obtain the final BLUP value.

The generalized heritability calculation formula is as follows: $h^{2}=\sigma g^{2} /\left(\sigma g^{2}+\sigma g e^{2} / e+\sigma \varepsilon^{2} / r e\right)[35]$, where $\sigma g^{2}$ represents genetic variance, $\sigma g e^{2}$ represents the genotype by environment interaction variance, $\sigma \varepsilon^{2}$ represents a residual error, and e and $\mathrm{r}$ represent the number of environments and the number of repetitions in each environment, respectively.

\section{Candidate gene prediction and function annotation}


To select independent and significant candidate genes, genes with $\mathrm{R}^{2}<0.2$ between significant SNPs sites on the same chromosome were considered to not be in the same segment. The specific physical location of SNPs refers to the v2 version of the maize B73 genome sequence. In the range of $50 \mathrm{~kb}$ upstream and downstream of the significantly related SNPs sites, genes that have been annotated or analyzed according to functional domains, such as genes in this segment or their homologous genes in Arabidopsis participating in root morphogenesis, were searched. Then, the gene was predicted as a candidate gene.

The B73 v2 versiB website. Functional annotations were performed on the selected maize BRA and BRD candidate genes based on the orthologous gene information of Arabidopsis and maize[36].

\section{Genome-wide association map and phenotypic variation contribution of important loci}

The original number and the obtained BLUP value were saved in tab delimiter format, and 558,629 SNPs markers (MAF $\geq 0.05$ ) were used for GWAS analysis from two genotyping platforms (RNA-seq and SNPs chip). The MLM was used to perform an association analysis on BRA and BRD[8] and to combine the influence of population structure (K) and kinship (Q) to remove false-positive results[37]. The method by Hao (2015) was used to count the number of independent SNPs with $\mathrm{R}^{2}<0.2$ in all LDs[38]. According to the Bonferroni correction threshold MLM model of the same association group published in the article, $P<1 / n(n=558,629)[36,39]$; therefore, the MLM model threshold of this research topic was $P<1.79 \times 10^{-6}$. The contribution rate of the SNPs phenotype was calculated using the R language "anova ()" function. After considering the population structure, the phenotypic contribution rate $\left(R^{2}\right)$ of each significant SNP site was calculated using the following linear model:

$$
\begin{gathered}
Y=\alpha X+\beta P+\varepsilon(1) \\
Y=\beta P+\varepsilon(2)
\end{gathered}
$$

The phenotypic contribution rate of all maize BRA- and BRD-related SNPs was calculated using the following linear model:

$$
\begin{gathered}
Y=\alpha \sum_{i=1}^{m} X i+\beta P+\varepsilon(3) \\
Y=\beta P+\varepsilon(2)
\end{gathered}
$$

In the model, $Y$ and $X$ represent phenotypic variables and SNPs genotype variables, respectively; $P$ is a subpopulation variable, $a$ is a SNP effect, $\beta$ is a subpopulation effect, and $\varepsilon$ is a random effect.

\section{Annotation of candidate genes}

The SNPs with the most significance within the same LD block $d\left(r^{2}<0.2\right)$ was selected to represent the locus. The physical locations of the SNPs were recorded according to the B73 RefGen_v2 (www.maizesequence.org). The corresponding genes were annotated by performing BLASTP search through NCBI website, and the candidate genes were assigned into different biological processes on the basis of the literatures describing the function of their homologs in other species or the knowledge in conserved domain database (CDD).

\section{Heat-map of candidate genes}

Raw datasets of RNA-Seq from different maize tissues were downloaded from NCBI's Sequence Read Archive (SRA) database. RNA-Seq reads were aligned to the maize B73 reference genome v2 (www.maizesequence.org) using the TopHat2 pipeline with the built-in Bowtie mapping program. The unique mapped reads were counted by htseq-count (HTSeq). To normalize the RNA-Seq data across the eleven samples from different maize tissues, we used the scaling normalization method provided in the edgeR package, based on a trimmed mean of M-values algorithm to compute the scaling factors according to the library size of each sample. After edgeR normalization, the RPKM values were averaged from replicates and used in the further analysis. The values used in the Fig. 6 are the $\log 10$ transformed ratio of normalized RPKM count in husk relative to other tissues. The values greater than +2 or less than -1 are adjusted to 2 or -1 , respectively.

\section{Additional files}

Additional file 1: Table S1.The phenotype data of 508 inbred lines were measured independently in Shenyang City, Liaoning Province, in 2016 and 2017 and Siping City, Jilin Province, in 2016, and the phenotypic values of three environmental field trials were used as random effects to calculate the BLUP values.

Additional file 2: Table S2.All candidate genes were divided into eight functional types: anabolic pathways, protein binding, hormone signaling pathways, transport regulation, DNA binding, cell transport, structural proteins, and unknown functions.

\section{Abbreviations}

BR: Brace root; BRA: Brace root angle; BRD: Brace root diameter; GWAS: Genome-wide association study; BLUP: Best linear unbiased prediction; CIMMYT: International Maize and Wheat Improvement Center; TN: Brace-root tier number; RN: Brace root number; RBR: Radius of the brace root; LD: Linkage disequilibrium; MATE: Multi-drug and toxin extrusion; $\mathrm{H}^{2}$ : Family mean-based broad-sense heritability; E: Environment; PH: Plant height; EH: Ear height; ELL: Ear position leaf length; TMAL: Tassel maximum axis length; TBN: Tassel branch number; LNAE: Leaf number above ear; DTA: Days to anthesis; DTS: Days to silking; DTH: Days to heading; EL: Ear length; ED: Ear diameter; CD: Cob diameter; KNPR: Kernel number per row; GW: Grain weight; CW: Cob weight; KW: Kernel width; Q matrix: Population structure; K matrix: Kinship relationship; $\mathrm{R}^{2}$ : Percentage of phenotypic variation explained by the identified SNPs 


\section{Declarations}

\section{Funding}

This research was supported by the Shanghai Agriculture Applied Technology Development Program, China(Grant No.Z20190101); the National Science Foundation for Young Scientists of China(31801442); the National Key Research and Development Program of China (Grand No. 2016YFD0101803). We are grateful to X. Yang (China Agricultural University) for providing seeds of the 508-line association panel.

\section{Availability of data and materials}

The datasets generated and analysed during the current study are deposited in [dbGaP] repository and accession numbers will be shared once they are available.The datasets used and analysed during the current study available from the corresponding author on reasonable request.

\section{Authors' contributions}

Daqiu Sun: Formal analysis, Software, Writing-original draft, Writing-review \& editing, Visualization. Sibo Chen: Data curation, Investigation. Zhenhai Cui: Supervision. Jingwei Lin: Supervision. Meiling Liu: Visualization. Yueting Jin: Validation. Ao Zhang: Validation. Yuan Gao: Validation. Huiying Cao: Conceptualization, Supervision. Yanye Ruan: Conceptualization.

\section{Ethics approval and consent to participate}

Among the plant materials used in this study, the genome-wide association population was preserved by College of Biological Science and Technology, Liaoning Province Research Center of Plant Genetic Engineering Technology, Shenyang Key Laboratory of Maize Genomic Selection Breeding, Shenyang Agricultural University. These plant materials don't include any wild species at risk of extinction. No specific permits are required for sample collection in this study. We comply with relevant institutional, national, and international guidelines and legislation for plant study.

\section{Competing interests}

The authors declare that they have no competing interests.

\section{Consent for publication}

Not applicable.

\section{Author details}

${ }^{1}$ College of Biological Science and Technology, Liaoning Province Research Center of Plant Genetic Engineering Technology, Shenyang Key Laboratory of Maize Genomic Selection Breeding, Shenyang Agricultural University, Shenyang, 110866, China. ${ }^{2}$ Rice Research Institute, Shenyang Agricultural University, Key Laboratory of Northern geng Super Rice Breeding, Ministry of Education, Shenyang 110866, China. *Corresponding authors.

\section{References}

1. Ju C, Zhang W, Liu Y, Gao Y, Wang X, Yan J, Yang X, Li J. Genetic analysis of seedling root traits reveals the association of root trait with other agronomic traits in maize. BMC Plant Biol. 2018; 18(1):171. doi: 10.1186/s12870-018-1383-5.

2. Zhang Z, Zhang X, Lin Z, Wang J, Xu M, Lai J, Yu J, Lin Z. The genetic architecture of nodal root number in maize. Plant J. 2018; 93(6):1032-1044. doi: $10.1111 /$ tpj.13828.

3. Varney, G. T., and M. J. Canny, Rates of water uptake into the mature root system of maize plants. New Phytologist. 1993; 775-786. doi: 10.1111/j.14698137.1993.tb03789.x.

4. Aiken RM, Smucker AJ. Root system regulation of whole plant growth. Annu Rev Phytopathol. 1996; 34:325-46. doi: 10.1146/annurev.phyto.34.1.325.

5. Hochholdinger F, Tuberosa R. Genetic and genomic dissection of maize root development and architecture. Curr Opin Plant Biol. 2009; 12(2):172-7. doi: 10.1016/j.pbi.2008.12.002.

6. Li YJ, Fu YR, Huang JG, Wu CA, Zheng CC. Transcript profiling during the early development of the maize brace root via Solexa sequencing. FEBS J. 2011; 278(1):156-66. doi: 10.1111/j.1742-4658.2010.07941.x.

7. LX Ku,ZH Sun,CL Wang,J Zhang,RF Zhao,HY Liu,GQ Tai,YH Chen. QTL mapping and epistasis analysis of brace root traits in maize. Molecular breeding. 2012; 30(2), 697-708. doi: 10.1007/s11032-011-9655-X.

8. Liu P, Yan K, Lei YX, Xu R, Zhang YM, Yang GD, Huang JG, Wu CA, Zheng CC. Transcript profiling of microRNAs during the early development of the maize brace root via Solexa sequencing. Genomics. 2013; 101(2):149-56. doi: 10.1016/j.ygeno.2012.11.004.

9. Pace J, Gardner C, Romay C, Ganapathysubramanian B, Lübberstedt T. Genome-wide association analysis of seedling root development in maize (Zea mays L.). BMC Genomics. 2015; 5;16(1):47. doi: 10.1186/s12864-015-1226-9.

10. JL Bennetzen, SC Hake.The maize root system: morphology, anatomy, and genetics. In Handbook of maize: Its biology (pp. 145-160). Springer. 2009; doi: 10.1007/978-0-387-79418-1_8.

11. Lynch, J. P. Roots of the second green revolution. Australian Journal of Botany. 2007; 55(5), 493-512. doi: 10.1071/BT06118. 
12. Cui Z, Xia A, Zhang A, Luo J, Yang X, Zhang L, Ruan Y, He Y. Linkage mapping combined with association analysis reveals QTL and candidate genes for three husk traits in maize. Theor Appl Genet. 2018; 131(10):2131-2144. doi: 10.1007/s00122-018-3142-2.

13. Gu, D., Mei, X., Yu, T., Sun, N., Xu, D. E., Liu, C., \& Cai, Y. QTL identification for brace-root traits of maize in different generations and environments. Crop Science. 2017; 57(1), 13-21. doi: 10.2135/cropsci2016.01.0031.

14. Lynch J. Root Architecture and Plant Productivity. Plant Physiol. 1995; 109(1):7-13. doi: 10.1104/pp.109.1.7.

15. Li P, Chen F, Cai H, Liu J, Pan Q, Liu Z, Gu R, Mi G, Zhang F, Yuan L. A genetic relationship between nitrogen use efficiency and seedling root traits in maize as revealed by QTL analysis. J Exp Bot. 2015; 66(11):3175-88. doi: 10.1093/jxb/erv127.

16. Zuber M S. Evaluations of maize root systems under various environments Proc. 23rd Corn and Sorghum Res. Conf. 1968.

17. Duparque A, Pellerin S. Some morphological characters of maize root and shoot systems and their relationships with resistance to root lodging. 1990.

18. Stamp P, Kiel C. Seedling traits of maize as indicators of root lodging. Agronomie. 1992; 12(2): 157-162. doi: 10.1051/agro:19920203.

19. Crook M J, Ennos A R. Stem and root characteristics associated with lodging resistance in four winter wheat cultivars. The Journal of Agricultural Science. 1994; 123(2): 167-174. doi: 10.1017/S0021859600068428.

20. Stokes A, Ball J, Fitter A H, et al. An experimental investigation of the resistance of model root systems to uprooting. Annals of Botany. 1996; 78(4): 415421. doi: 10.1016/S0142-9612(01)00350-7.

21. Mollier A, Pellerin S. Maize root system growth and development as influenced by phosphorus deficiency[J]. Journal of Experimental Botany. 1999; 50(333): 487-497.

22. Hermans C, Hammond JP, White PJ, Verbruggen N. How do plants respond to nutrient shortage by biomass allocation Trends Plant Sci. 2006; 11(12):610-7. doi: 10.1016/j.tplants.2006.10.007.

23. Silberbush M., Barber,. Sensitivity of simulated phosphorus uptake to parameters used by a mechanistic-mathematical model. Plant and Soil. 1983; 74(1), 93-100.

24. Zhu J, Lynch JP. The contribution of lateral rooting to phosphorus acquisition efficiency in maize (Zea mays) seedlings. Funct Plant Biol. 2004; 31(10):949-958. doi: 10.1071/FP04046.

25. Li J, Chen F, Li Y, Li P, Wang Y, Mi G, Yuan L. ZmRAP2.7, an AP2 Transcription Factor, Is Involved in Maize Brace Roots Development. Front Plant Sci. 2019; 4;10:820. doi: 10.3389/fpls.2019.00820.

26. Suzuki M, Sato Y, Wu S, Kang BH, McCarty DR. Conserved Functions of the MATE Transporter big embryo1 in Regulation of Lateral Organ Size and Initiation Rate. Plant Cell. 2015; 27(8):2288-300. doi: 10.1105/tpc.15.00290.

27. Yu J, Buckler ES. Genetic association mapping and genome organization of maize. Curr Opin Biotechnol. 2006; 17(2):155-60. doi: 10.1016/j.copbio.2006.02.003.

28. Atwell S, Huang YS, Vilhjálmsson BJ, Willems G, Horton M, Li Y, Meng D, Platt A, Tarone AM, Hu TT, Jiang R, Muliyati NW, Zhang X, Amer MA, Baxter I, Brachi B, Chory J, Dean C, Debieu M, de Meaux J, Ecker JR, Faure N, Kniskern JM, Jones JD, Michael T, Nemri A, Roux F, Salt DE, Tang C, Todesco M, Traw MB, Weigel D, Marjoram P, Borevitz JO, Bergelson J, Nordborg M. Genome-wide association study of 107 phenotypes in Arabidopsis thaliana inbred lines. Nature. 2010; 3;465(7298):627-31. doi: 10.1038/nature08800.

29. Huang X, Zhao Y, Wei X, Li C, Wang A, Zhao Q, Li W, Guo Y, Deng L, Zhu C, Fan D, Lu Y, Weng Q, Liu K, Zhou T, Jing Y, Si L, Dong G, Huang T, Lu T, Feng Q, Qian Q, Li J, Han B. Genome-wide association study of flowering time and grain yield traits in a worldwide collection of rice germplasm. Nat Genet. 2011; 4;44(1):32-9. doi: 10.1038/ng.1018.

30. Riedelsheimer C, Lisec J, Czedik-Eysenberg A, Sulpice R, Flis A, Grieder C, Altmann T, Stitt M, Willmitzer L, Melchinger AE. Genome-wide association mapping of leaf metabolic profiles for dissecting complex traits in maize. Proc Natl Acad Sci USA. 2012; 5;109(23):8872-7. doi: 10.1073/pnas.1120813109.

31. Aranzana MJ, Kim S, Zhao K, Bakker E, Horton M, Jakob K, Lister C, Molitor J, Shindo C, Tang C, Toomajian C, Traw B, Zheng H, Bergelson J, Dean C, Marjoram P, Nordborg M. Genome-wide association mapping in Arabidopsis identifies previously known flowering time and pathogen resistance genes. PLoS Genet. 2005; 1(5):e60. doi: 10.1371/journal.pgen.0010060.

32. Cui Z, Luo J, Qi C, Ruan Y, Li J, Zhang A, Yang X, He Y. Genome-wide association study (GWAS) reveals the genetic architecture of four husk traits in maize. BMC Genomics. 2016; 21;17(1):946. doi: 10.1186/s12864-016-3229-6.

33. X Yang,S Gao,S Xu,Z Zhang,BM Prasanna,L Lin,J Li,J Yan.Characterization of a global germplasm collection and its potential utilization for analysis of complex quantitative traits in maize. Molecular Breeding. 2011; 28(4), 511-526. doi: 10.1007/s11032-010-9500-7.

34. Li Q, Yang X, Xu S, Cai Y, Zhang D, Han Y, Li L, Zhang Z, Gao S, Li J, Yan J. Genome-wide association studies identified three independent polymorphisms associated with a-tocopherol content in maize kernels. PLoS One. 2012; 7(5):e36807. doi: 10.1371/journal.pone.0036807.

35. Knapp SJ. Confidence intervals for heritability for two-factor mating design single environment linear models. Theor Appl Genet. 1986; 72(5):587-91. doi: 10.1007/BF00288995. PMID: 24248067.

36. Li H, Peng Z, Yang X, Wang W, Fu J, Wang J, Han Y, Chai Y, Guo T, Yang N, Liu J, Warburton ML, Cheng Y, Hao X, Zhang P, Zhao J, Liu Y, Wang G, Li J, Yan J. Genome-wide association study dissects the genetic architecture of oil biosynthesis in maize kernels. Nat Genet. 2013; 45(1):43-50. doi: $10.1038 / \mathrm{ng} .2484$

37. BA Loiselle,VL Sork,J Nason,C Graham. Spatial genetic structure of a tropical understory shrub, Psychotria officinalis (Rubiaceae). American journal of botany. 1995; 82(11): 1420-1425. doi: 10.1007/s10666-007-9130-6.

38. Hao D, Zhang Z, Cheng Y, Chen G, Lu H, Mao Y, Shi M, Huang X, Zhou G, Xue L. Identification of Genetic Differentiation between Waxy and Common Maize by SNP Genotyping. PLoS One. 2015; 13;10(11):e0142585. doi: 10.1371/journal.pone.0142585.

Page 9/13 
39. Yang N, Lu Y, Yang X, Huang J, Zhou Y, Ali F, Wen W, Liu J, Li J, Yan J. Genome wide association studies using a new nonparametric model reveal the genetic architecture of 17 agronomic traits in an enlarged maize association panel. PLoS Genet. 2014; 11;10(9):e1004573. doi:

10.1371/journal.pgen.1004573.

40. Ao Z, Zhenhai C, Cong L. Identification of maize brace-root quantitative trait loci in are combinant in bred line population. Euphytica. 2018 ; $214(9): 168$. doi: 10.1007/s10681-018-2203-6.

41. Phuthaworn C, Nguyen NH, Quinn J, Knibb W. Moderate heritability of hepatopancreatic parvovirus titre suggests a new option for selection against viral diseases in banana shrimp (Fenneropenaeus merguiensis) and other aquaculture species. Genet Sel Evol. 2016; 7;48(1):64. doi: 10.1186/s12711-0160243-8.

42. XML A, WRG A, CFL B, LA Jing, WA Shi. Effects of nitrogen fertilizer and chemical regulation on spring maize lodging characteristics, grain filling and yield formation under high planting density in Heilongjiang Province, China. Journal of Integrative Agriculture. 2021; 20(2):511-526. doi: 10.1016/S20953119(20)63403-7.

43. S Trachsel, SM Kaeppler, KM Brown, JP Lynch. Maize root growth angles become steeper under low N conditions. Field Crops Research. 2013; 140: 1831. doi: 10.1016/j.fcr.2012.09.010.

44. Szulc P. Improvement of the effectiveness of maize (Zea mays L.) fertilization with nitrogen by the application of magnesium part III. Quality of ears designed for silage ccm. Acta Scientiarum Polonorum Agricultura. 2009; 8(3): 37.

45. Nitrogen. Reports from University of Missouri Highlight Recent Findings in Nitrogen (Pre-and Post-silking Carbohydrate Concentrations in Maize Earleaves and Developing Ears in Response to Nitrogen Availability). Chemicals \& Chemistry. 2016.

46. Wei S, Wang X, Li G, Qin Y, Jiang D, Dong S. Plant Density and Nitrogen Supply Affect the Grain-Filling Parameters of Maize Kernels Located in Different Ear Positions. Front Plant Sci. 2019; 1;10:180. doi: 10.3389/fpls.2019.00180.

47. Raihan M, Liu J, Huang J, Guo H, Pan Q, Yan J. Multi-environment QTL analysis of grain morphology traits and fine mapping of a kernel-width QTL in Zheng58 × SK maize population. Theoretical \& Applied Genetics. 2016; 129(8):1465-1477. doi: 10.1007/s00122-016-2717-z.

48. Camus-Kulandaivelu L, Veyrieras JB, Madur D, Combes V, Fourmann M, Barraud S, Dubreuil P, Gouesnard B, Manicacci D, Charcosset A. Maize adaptation to temperate climate: relationship between population structure and polymorphism in the Dwarf8 gene. Genetics. 2006; 172(4):2449-63. doi: 10.1534/genetics.105.048603.

49. De Dorlodot S, Forster B, Pagès L, Price A, Tuberosa R, Draye X. Root system architecture: opportunities and constraints for genetic improvement of crops. Trends Plant Sci. 2007; 12(10):474-81. doi: 10.1016/j.tplants.

50. Xu SL, Rahman A, Baskin TI, Kieber JJ. Two leucine-rich repeat receptor kinases mediate signaling, linking cell wall biosynthesis and ACC synthase in Arabidopsis. Plant Cell. 2008; 20(11):3065-79. doi: 10.1105/tpc.108.063354.

51. Basu D, Tian L, Debrosse T, Poirier E, Emch K, Herock H, Travers A, Showalter AM. Glycosylation of a Fasciclin-Like Arabinogalactan-Protein (SOS5) Mediates Root Growth and Seed Mucilage Adherence via a Cell Wall Receptor-Like Kinase (FEI1/FEI2) Pathway in Arabidopsis. PLoS One. 2016; 5;11(1):e0145092. doi: 10.1371/journal.pone.0145092.

52. Swarup R, Friml J, Marchant A, Ljung K, Sandberg G, Palme K, Bennett M. Localization of the auxin permease AUX1 suggests two functionally distinct hormone transport pathways operate in the Arabidopsis root apex. Genes Dev. 2001; 15;15(20):2648-53. doi: 10.1101/gad.210501.

53. Muday GK, Murphy AS. An emerging model of auxin transport regulation. Plant Cell. 2002; 14(2):293-9. doi: 10.1105/tpc.140230.

54. Michniewicz M, Zago MK, Abas L, Weijers D, Schweighofer A, Meskiene I, Heisler MG, Ohno C, Zhang J, Huang F, Schwab R, Weigel D, Meyerowitz EM, Luschnig C, Offringa R, Friml J. Antagonistic regulation of PIN phosphorylation by PP2A and PINOID directs auxin flux. Cell. 2007; 21;130(6):1044-56. doi: 10.1016/j.cell.2007.07.033.

55. Rashotte AM, DeLong A, Muday GK. Genetic and chemical reductions in protein phosphatase activity alter auxin transport, gravity response, and lateral root growth. Plant Cell. 2001; 13(7):1683-97. doi: 10.1105/tpc.010158.

56. Zhan X, Wang B, Li H, Liu R, Kalia RK, Zhu JK, Chinnusamy V. Arabidopsis proline-rich protein important for development and abiotic stress tolerance is involved in microRNA biogenesis. Proc Natl Acad Sci USA. 2012; 30;109(44):18198-203. doi: 10.1073/pnas.1216199109.

57. Remy E, Cabrito TR, Baster P, Batista RA, Teixeira MC, Friml J, Sá-Correia I, Duque P. A major facilitator superfamily transporter plays a dual role in polar auxin transport and drought stress tolerance in Arabidopsis. Plant Cell. 2013; 25(3):901-26. doi: 10.1105/tpc.113.110353.

58. Woodward AW, Bartel B. Auxin: regulation, action, and interaction. Ann Bot. 2005; 95(5):707-35. doi: 10.1093/aob/mci083.

59. Sheldrake P. Carrier-mediated Auxin Transport. Planta. 1974; 118(2):101-121. doi: 10.2307/23370985.

60. Goldsmith M H M. The Polar Transport of Auxin. Annual Review of Plant Physiology. 2003; 28(1):439-478. doi: 10.1146/annurev.pp.28.060177.002255.

61. Fu X, Harberd NP. Auxin promotes Arabidopsis root growth by modulating gibberellin response. Nature. 2003; 13;421(6924):740-3. doi: 10.1038 /nature01387.

62. Peng Y, Chen L, Lu Y, Ma W, Tong Y, Li Y. DAR2 acts as an important node connecting cytokinin, auxin, SHY2 and PLT1/2 in root meristem size control. Plant Signal Behav. 2013; 8(6):e24226. doi: 10.4161/psb.24226.

63. Dello loio R, Nakamura K, Moubayidin L, Perilli S,Taniguchi M, Morita MT. A genetic frameworkfor the control of cell division and differentiationin the root meristem. Science. 2008; 322:1380-4. doi: org/10.1126/science.1164147.

\section{Figures}




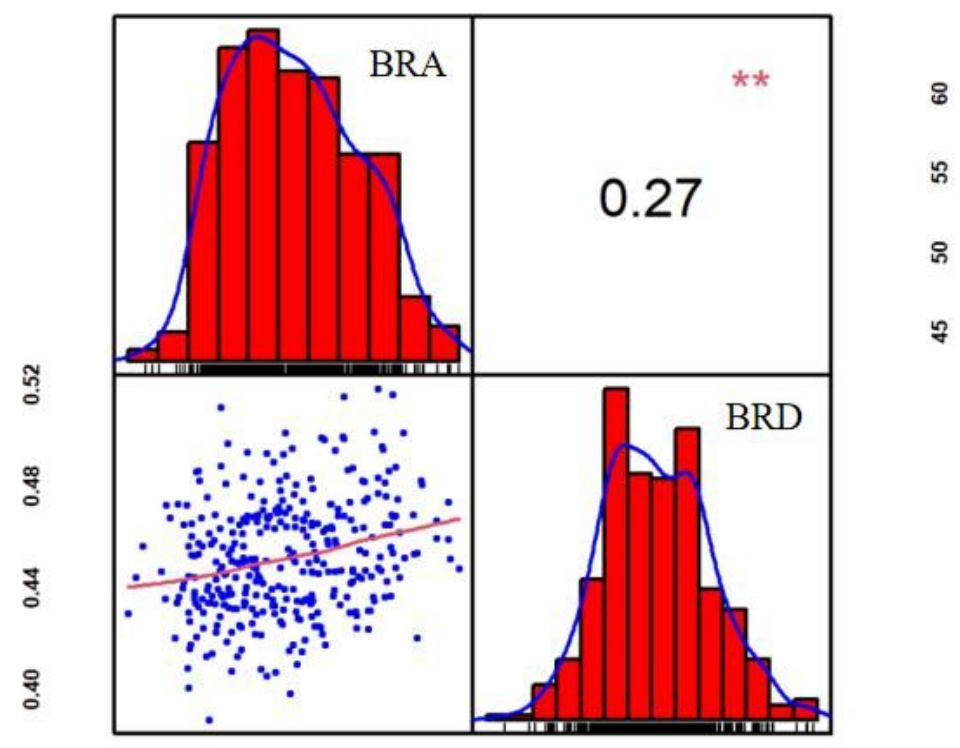

$45 \quad 50 \quad 55 \quad 60$

Figure 1

Correlation of BRA and BRD traits. Frequency distribution and correlation of BRA and BRD measured in 16SY (2016 Shenyang), 16SP (2016 Siping), 17SY (2017 Shenyang), and BLUPs (best unbiased linear predictive values). The graphs on the diagonal represent the phenotypic distribution frequency of BLUPs in BRA and BRD. The value above the diagonal is the Pearson correlation coefficient between the two traits. Below the diagonal is a scatter plot of the two traits.

* represents a significant difference at the 0.05 level; ** represents a very significant difference at the 0.01 level

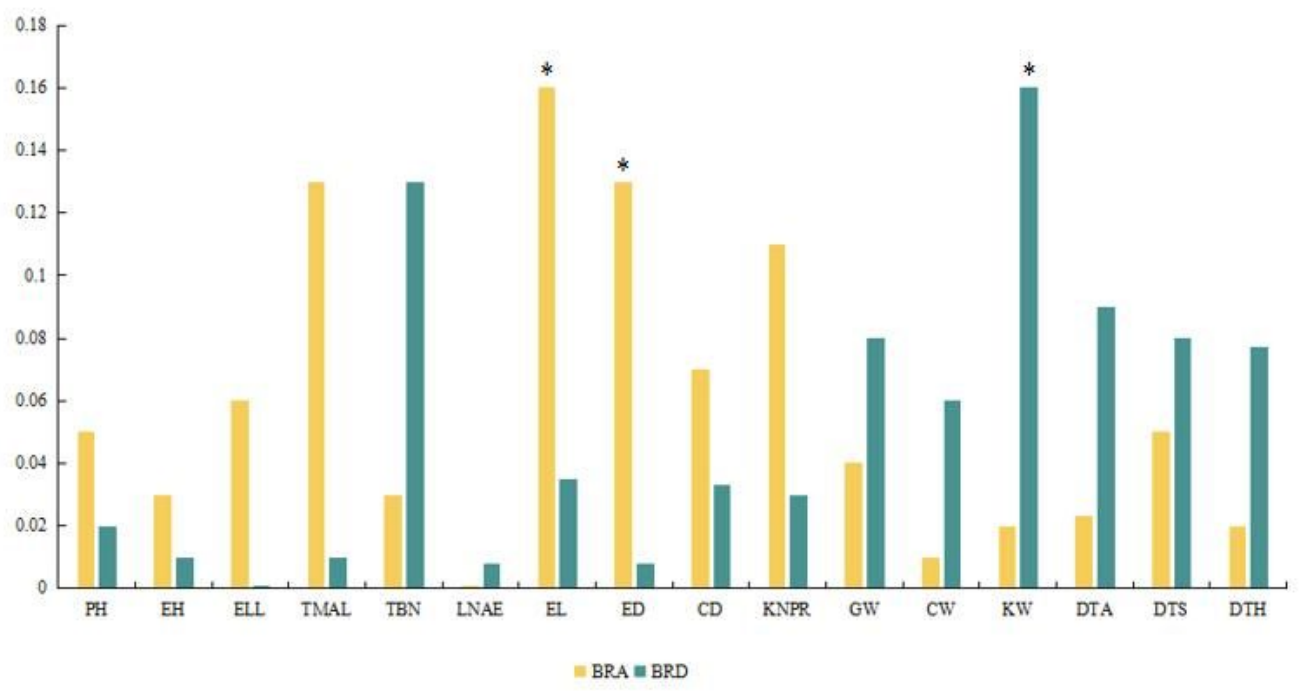

Figure 2

Correlation between brace root angle, diameter, and 16 other agronomic traits. *Significant when $\mathrm{P} \leq 0.05$; **Very significant when $\mathrm{P} \leq 0.01$ 

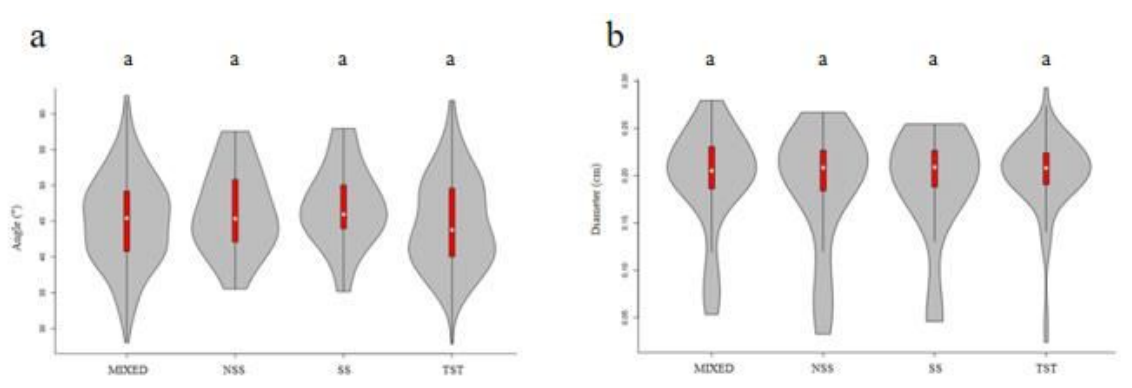

c

d
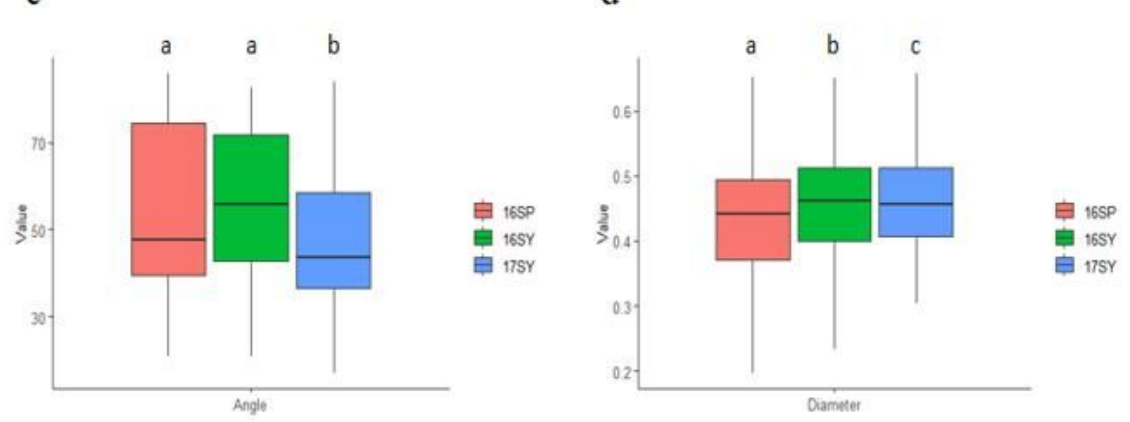

\section{Figure 3}

Violin view of brace root angle and diameter of the four major subgroups and different environments. Sub-figures a and b are violin diagrams of the BRA and BRD distribution of maize, respectively. Analysis of variance was used to examine the differences in traits between subgroups. For MIXED, NSS, SS, and TST, the self-intersection coefficients included in each subgroup were 215, 70, 27 and 196, respectively. Subgroups $\mathrm{c}$ and d are box plots of the BRA and BRD of maize, respectively. Analysis of variance was used to examine the differences in traits between environments

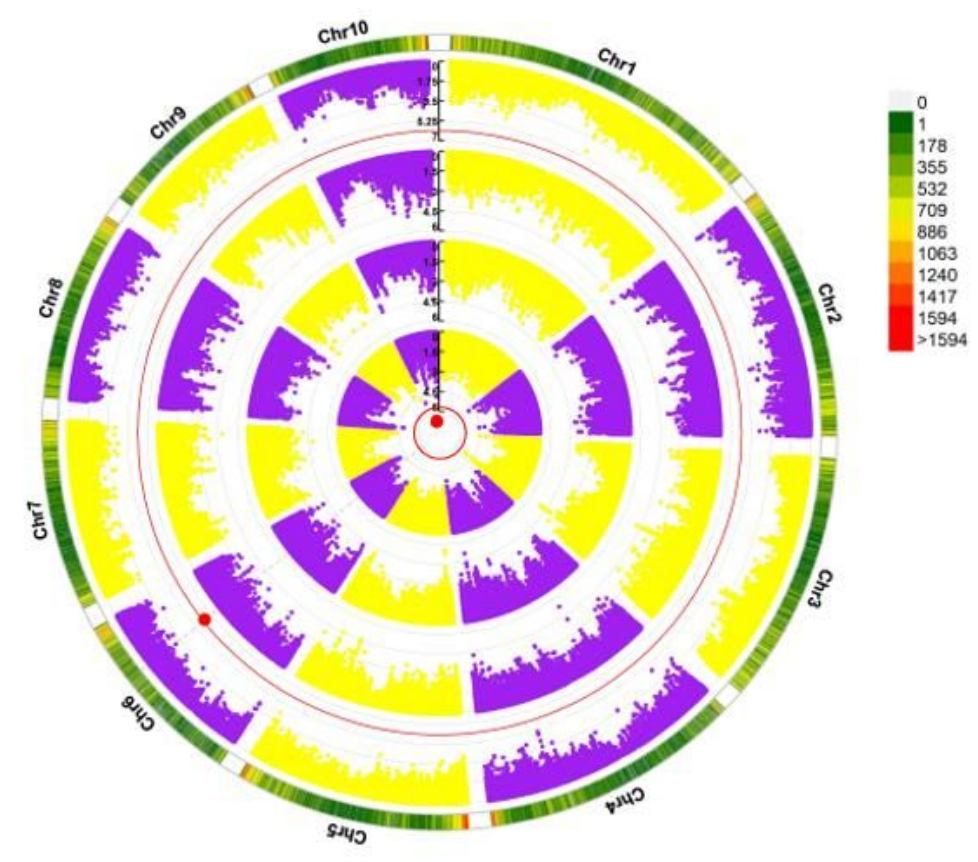

\section{Figure 4}

Manhattan circle graph derived from GWAS showing the results of using MLM and correlation mapping, the genome location, and the allelic effect of significant SNPs located near representative genes of BRA. Each dot represents an SNP. The red dots indicate significant P-values related to BRA. The red line in the circle represents the significant threshold of Bonferroni correction of 1.79×10-6. From the outside to the inside, the Manhattan circle chart is Angle, 16Angle-SY, 16-Angle-JL, and 17-Angle-SY 


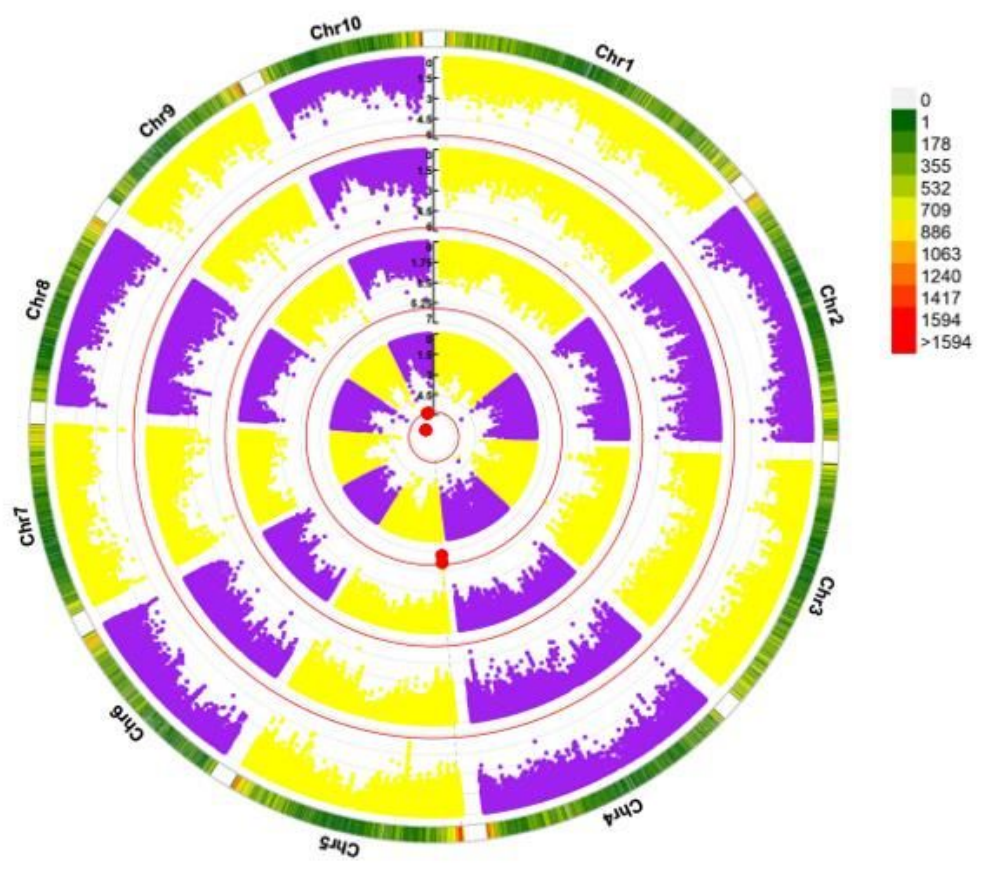

Figure 5

Manhattan circle graph derived from GWAS showing the results of using MLM and correlation mapping, the genome location, and the allelic effect of the significant SNPs located near the representative gene of BRD. Each dot represents an SNP. The red dots indicate significant P-values related to BRD. The red line in the circle represents the significant threshold of Bonferroni correction of 1.79×10-6. The Manhattan circle map is Diameter, 16-Diameter-SY, 16-

Diameter-JL, and 17-Diameter-SY from the outside to the inside

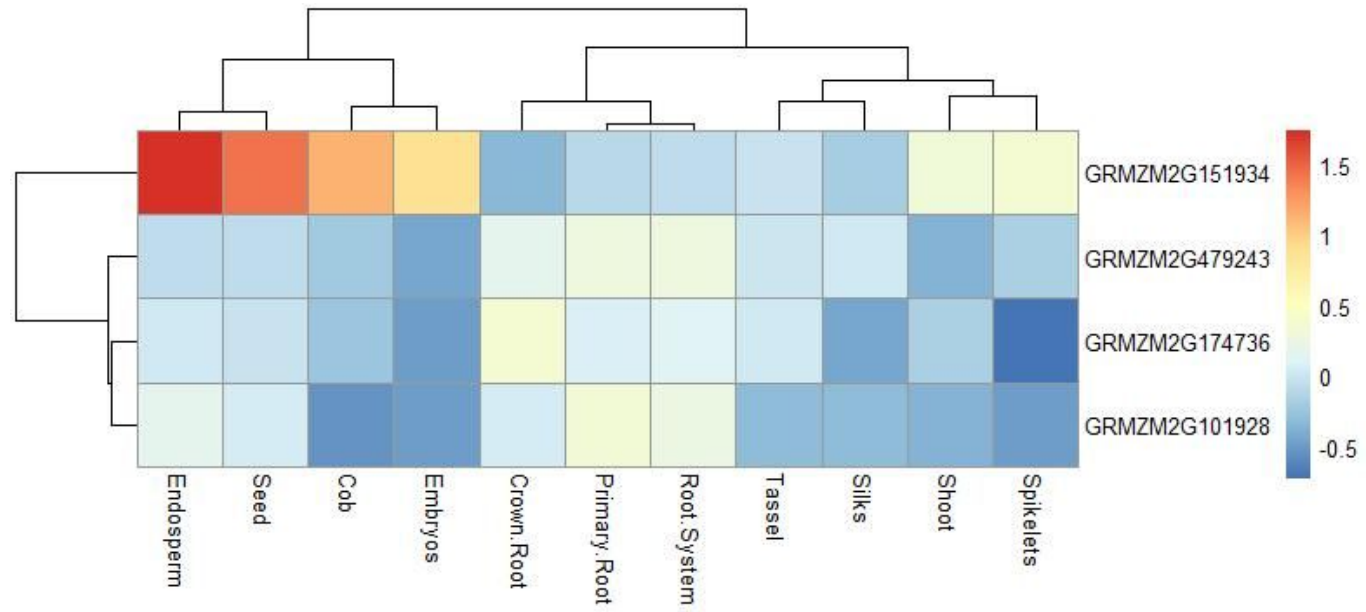

Figure 6

Candidate gene tissue-specific expression heatmap. The values used in the figure are the log10 conversion ratios of normalized PRKM counts in brace root tissues to those in other tissues, as shown at the bottom of each column. Columns and rows are sorted according to similarity (hierarchical cluster analysis on top and left). Compared with other tissues, red, yellow and blue represent higher, similar and lower specific genes in brace roots, respectively

\section{Supplementary Files}

This is a list of supplementary files associated with this preprint. Click to download.

- Supplementary1.xlsx

- Supplementary2.doc 